\title{
Contraste entre \\ los determinantes de la \\ inversión en I\&D \\ y del registro de patentes en sectores industriales de Colombia*
}

\section{Relationship between a firm's innovation strategy}

and its decision to patent: empirical evidence from
colombian manufacturing companies

Jorge Luis Juliao Rossi** Julia Andrea Pineda Acero**** Fernando Barrios Aguirre*****

* Artículo producto del proyecto de investigación los determinantes de la inversión en I\&D en Colombia financiado por la Universitaria Agustiniana, Uniagustiniana. Cómo citar este artículo: Juliao, J. L., Pineda, J. A., Barrios, F. (2015). Contraste entre los determinantes de la inversión en I\&D y del registro de patentes en sectores industriales de Colombia. Revista CIFE, 17(27), 185-208

** Docente investigador de la Facultad de Ciencias Económicas y Administrativas, Programa de Administración de Empresas, Universitaria Agustiniana. PhD en Administración, Universidad de los Andes. Correo para correspondencia: admonempresas@uniagustiniana.edu.co. Enviar correspondencia a Dirección del programa de Administración de Empresas, Universitaria Agustiniana, Avenida Ciudad De Cali No 11B - 95. Bogotá, Colombia. Teléfono: +(571) 4193200.

*** Docente e investigadora del Centro de Tecnologías para la Academia, Universidad de la Sabana, Chía, Colombia. M.Sc. en Administración, Universidad de los Andes. 
Recibido: 11 de febrero de 2015

Revisado: 23 de febrero de 2015

Aprobado: 2 de febrero de 2016

\section{Resumen}

Esta investigación contrasta los determinantes del esfuerzo innovador y del conteo de patentes de sectores industriales de una economía emergente. En muchos estudios se tiende a considerar el gasto en I\&D y el conteo de patentes como indicadores de desempeño innovador. Sin embargo, el presente trabajo prueba que el esfuerzo innovador y el registro de patentes son dos comportamientos diferentes, no directamente relacionados y sin los mismos determinantes. Se usan datos provenientes de la Tercera Encuesta de Desarrollo e Innovación Tecnológica EDIT-III (2005-2006), diseñada y aplicada por el DANE, el DNP y Colciencias. Los resultados sugieren que las condiciones del desarrollo tecnológico e institucional de Colombia se reflejan en relaciones entre determinantes y los dos indicadores de desempeño innovador, distintas de las que se observan en países industrializados.

Palabras clave: Sectores industriales, investigación y desarrollo (I\&D), patentes.

Clasificación JEL: O32, O34

\section{Abstract}

In this research are contrasted the determinants of $\mathrm{R} \& \mathrm{D}$ investment and patent count of industries belonging to an emerging country. In many studies is common to consider indistinctly these indicators as a proxy of innovation performance. However, this research shows that $\mathrm{R} \& \mathrm{D}$ investment and patent count are two different behaviors: these don not have necessarily a direct relationship and the same determinants. It was used de data provided for third survey of Innovation and Technology 2005-2006. Development (in forward EDIT) designed and applied bay Dane-DNP-Colciencias). The results obtained suggest that the institutional and technological conditions of Colombia are reflected in some relationships between innovation determinants and both innovation performance indicators used different from that observed in industrialized countries.

Keywords: Industry sectors, research and development $(\mathrm{R} \& \mathrm{D})$, patents.

Classification JEL: O32, O34 


\section{Introducción}

El gasto en I\&D y el registro de patentes han sido utilizados con frecuencia como medidas del desempeño innovador de empresas (Becheikh, Landry y Amara, 2006), sectores y países (De Rassenfosse y De la Potterie, 2009). Algunos investigadores han explorado la relación entre estos dos indicadores y han concluido que la inversión en I\&D es un insumo, mientras que el conteo de patentes es un resultado del proceso de innovación (Geisler, 2002). Las investigaciones de Duguet y Kabla (1998) y Brouwer y Kleinknecht (1999), entre otras, confirman una estrecha relación entre gasto en I\&D y registro de patentes en estudios transversales. Sin embargo, una reciente investigación longitudinal muestra que esta relación es mucho más débil de lo que anunciaban los estudios anteriores (Danguy, De Rassenfosse y De la Potterie, 2009).

A pesar de aceptarse una distinción conceptual entre inversión en I\&D y registro de patentes, cuando estos indicadores son usados como proxies de desempeño innovador, generalmente se proponen las mismas variables predictoras (Becheikh, Landry y Amara, 2006). En el presente artículo se confronta esa visión y se demuestra que las variables generalmente usadas para predecir estos dos indicadores de innovación los afectan de manera diferente y a veces opuesta. Se dilucida, además, cómo el contexto de desarrollo institucional y tecnológico característico de países emergentes acentúa tal contraste, hasta tal punto que los resultados difieren de la evidencia observada en países desarrollados. Para el logro del anterior fin, se usan datos provenientes de la Tercera Encuesta de Desarrollo e Innovación Tecnológica EDIT-III (2005-2006) - realizada por el DANE, el DNP y Colciencias- ${ }^{-}$se agregan a nivel sectorial. Con base en una muestra final de 53 sectores, se comparan los resultados de una regresión de Poisson - para la variable dependiente conteo de patentes- y una regresión lineal múltiple - para la variable dependiente promedio de inversión en I\&D-.

Se ha observado recientemente un acelerado interés por estudiar el fenómeno de la innovación en países latinoamericanos (Lundvall, Joseph, Chaminade y Vang, 2009). No obstante, es importante resaltar que aún se consideran pocas las investigaciones econométricas sobre determinantes del registro de patentes (v.gr. López y Orlicki, 2009) o de la inversión en I\&D (v.gr. Langebaek y Vásquez, 2007). Además, no se encontró una investigación sobre estos países en la cual se indague sobre las características de la relación entre estos dos indicadores y sus determinantes.

Los resultados obtenidos tienen importantes implicaciones para el diseño de políticas públicas en innovación. Las relaciones obtenidas entre los dos indicadores de desempeño usados como variables dependientes y las variables explicativas seleccionadas (inversión extranjera directa, fuentes externas de innovación y concentración de la innovación) se constituyen en una guía para la diferenciación de las estrategias gubernamentales dirigidas a estimular el gasto en I\&D de aquellas que incentivan el registro de patentes. Esta 
diferenciación es importante si se tiene en cuenta que la inversión en I\&D y el conteo de patentes son indicadores de progreso y cambio tecnológico en el ámbito industrial y a nivel nacional (Geisler, 2002).

El resto de la investigación está estructurado en cinco secciones. En la primera parte se presenta una revisión de la literatura con el fin de formular las hipótesis de investigación. En el segundo acápite se explican las características principales de los datos, las variables y los modelos estadísticos seleccionados. En la tercera sección se presentan los resultados de las estimaciones econométricas. En la cuarta parte se discuten los resultados a la luz de hallazgos de investigaciones relacionadas y del contexto colombiano. Las conclusiones se incluyen en el quinto apartado.

\section{Revisión de la literatura}

En la presente investigación, se ha optado por un análisis a nivel de sectores por tres razones. La primera es que existe un conjunto de teorías (e.g. patrones de innovación industrial, regímenes de innovación, sistemas de innovación) que coinciden en proponer que es posible identificar patrones de innovación seguidos por agregados de empresas, esto de acuerdo con las condiciones contextuales particulares (tecnológicas, institucionales, etc.) proporcionadas por su ubicación dentro de una región, industria o clúster. Dentro de estos enfoques teóricos se destacan las propuestas de sistemas nacionales (Lundvall, 2007) y sectoriales de innovación (Malerba, 2007). La idea subyacente a los estudios sectoriales de innovación es que las empresas dentro de un sector comparten propiedades generales de innovación, independientemente de la variedad de comportamientos individuales que pueden ser identificados entre firmas (Marsili, 1999). Al respecto, Malerba (2002) argumenta que firmas heterogéneas que enfrentan tecnologías afines, que investigan en torno a un mismo conocimiento base y que están embebidas en el mismo entorno institucional comparten rasgos comunes de comportamiento, aprendizaje y organización.

La segunda razón de la escogencia de los sectores como unidad de análisis es que consideramos que los resultados obtenidos a nivel sectorial pueden ser insumos importantes para el diseño de políticas de apoyo a la innovación.

La última razón obedece a que la mayoría de las firmas del sector manufacturero del país no tiene registro de patentes (DANE-DNP-Colciencias, 2011), en consecuencia, la agregación de datos a nivel sectorial permite la construcción de modelos estadísticos de mayor sencillez y parsimonia (Lutero, 2010).

Por otra parte, y con respecto a las variables explicativas incluidas, estas fueron seleccionadas con base en una revisión selectiva de la literatura acerca de los determinantes organizacionales y contextuales de desempeño innovador, literatura en la que se destacan 
los trabajos llevados a cabo por Damanpour y Aravind (2006) y Becheikh, Landry y Amara (2006). Específicamente, las variables explicativas en las cuales se centra esta investigación son capital extranjero, fuentes externas de ideas para la innovación y concentración de la inversión sectorial en innovación. Además, el análisis desarrollado se apoya en las siguientes variables de control: fuentes externas de financiamiento de la innovación, oportunidad tecnológica y grado educativo promedio del sector ${ }^{1}$.

\subsection{Propiedad extranjera}

El grado de participación que posea una empresa multinacional (EMN) en la propiedad de una subsidiaria se reconoce como un factor que aumenta la posibilidad de que la primera haga inversiones de I\&D. A mayor participación en la propiedad de la subsidiaria, menores serán los costos de transacción en que probablemente incurrirá la EMN para apropiarse de los conocimientos derivados de actividades de I\&D llevadas a cabo en estas (Gomes-Cassares, 1989). En este sentido, Feinberg y Gupta (2004) proponen que, en un contexto de propiedad compartida, las EMN estarán parcialmente restringidas por los intereses de apropiación del conocimiento generado en la filial por parte de los otros propietarios. Estos autores encuentran evidencia empírica que indica la existencia de una relación estadística positiva entre el grado de participación en la propiedad de la subsidiaria que posea la EMN y la probabilidad de que esta asigne responsabilidades de desarrollo de actividades de I\&D a la subsidiaria. Los anteriores resultados son coherentes con los hallazgos empíricos pioneros de Gatignon y Anderson (1988).

Acorde con los resultados empíricos reportados, parte de la literatura acerca de propiedad extranjera e innovación propone la existencia de una relación positiva entre la inversión extranjera directa y el desempeño innovador de las firmas (Singh, 2007). Sin embargo, estudios recientes muestran que esta relación depende de la decisión estratégica de las firmas extranjeras relacionada con la internacionalización de procesos completos de actividades de I\&D en sus subsidiarias (Cantwell y Molero, 2003; Cantwell y Piscitello, 2005; Molero y García, 2008).

La decisión de descentralización de actividades de I\&D e innovación por parte de las EMN se relaciona con la posibilidad de que estas firmas desarrollen un conjunto de competencias tecnológicas de nivel mundial asociadas con actividades de I\&D (Marín y Arza, 2009). Al respecto, se han identificado tres condiciones del país anfitrión como factores críticos para dichas actividades: i) la disponibilidad abundante de recurso humano cualificado (Molero y García, 2008); ii) la existencia de facilidades para la investigación (Cantwell y Molero, 2003; Molero y García, 2008); y iii) la fortaleza institucional, principalmente la protección de los derechos de propiedad intelectual o un régimen

1 Otra variable de control incluida fue la apertura exportadora del sector. No obstante, debido a problemas de ausencia de información relacionada con el cálculo de esta variable para trece sectores, se excluyó del estudio. 
adecuado de apropiación (Narula y Zanfei, 2005) que proteja a las EMN del riesgo potencial de que la tecnología de la empresa sea imitada por competidores (Singh, 2007).

En el contexto nacional, acorde con un estudio hecho por el Institute for Management Development (2010), la industria colombiana exhibe un bajo nivel de productividad (puesto 48 entre 59 países). Además, las empresas del país demuestran un nivel bajo de eficiencia (puesto 49). De acuerdo con los anteriores resultados, es poco probable que en promedio se evidencie el desarrollo por parte de las firmas locales de capacidades de I\&D de las cuales dependan las EMN.

Adicionalmente, según los resultados del Global Competitiviness Report 2010-2011 (Schwab, 2010), el sistema de educación superior y de cualificación del recurso humano del país se ha clasificado como de rango medio (puesto 69 entre 138 países) y la calidad de la educación en matemática y en ciencia es baja (lugar 93); lo anterior se refleja en una baja disponibilidad de científicos e ingenieros (puesto 88). Las relaciones universidadempresa se constituyen en una fortaleza del país (puesto 47); no obstante, dada la baja calidad de las instituciones de investigación científica (lugar 81), se puede aseverar que esta es una ventaja potencial y que actualmente no constituye una fuente de spillover atractivo para las EMN. Finalmente, este estudio revela un bajo nivel de gasto en I\&D del sector privado (puesto 79) y clasifica a Colombia entre los países con regímenes de propiedad intelectual débiles (lugar 93).

En síntesis, aunque el grado de propiedad que posean la EMN sobre la subsidiaria se asocia con una mayor delegación de actividades de I\&D, la evaluación de Colombia respecto a los elementos que hacen atractivo a un país como receptor de este tipo de actividades indica que este país no es un destino favorable para ello. Adicionalmente, el débil desarrollo de capacidades de I\&D por parte de las empresas locales no se constituye en un incentivo para que las EMN hagan inversiones locales en I\&D.

El comportamiento de las EMN en relación con el registro de patentes contrasta con lo anteriormente expuesto para la inversión en I\&D. El registro de patentes es gobernado principalmente por la decisión estratégica de las multinacionales de proteger localmente los derechos de propiedad de los inventos (o modificaciones de estos) generados en la casa matriz o en sus redes de I\&D descentralizadas; estos inventos generalmente han sido patentados previamente en el país de origen o en otros países de interés para la EMN (López y Orlicki, 2009). Los hallazgos empíricos de Aboites (2003) demuestran que la dinámica de registro de patentes en países latinoamericanos es liderada por empresas no residentes, tratándose en su mayoría de patentes no desarrolladas localmente.

Con base en los argumentos expuestos, se espera probar que: 
Hipótesis 1: 1a) existe una relación negativa entre el grado de propiedad extranjera del sector y la inversión promedio en IED por sector y $1 \mathrm{~b}$ ) existe una relación positiva entre el grado de propiedad extranjera del sector y el número de patentes obtenidas por sector.

\subsection{Fuentes externas de ideas para la innovación}

Los procesos de innovación son el resultado natural de interacciones sociales recurrentes (redes) en las cuales diversos actores comparten conocimientos complementarios (Blomqvist y Levy, 2006). Lundvall (2007) propone que la innovación no es un proceso aislado, sino el resultado de un sistema constituido por las interacciones entre firmas, clientes, proveedores, competidores, universidades, centros de investigación y organizaciones públicas y privadas. Se ha demostrado que estas redes le permiten a la empresa superar las deficiencias en la información, el conocimiento científico, los recursos y competencias (Romijn y Albaladejo, 2002). Acorde con lo anterior, se ha evidenciado una relación consistente y positiva entre desempeño innovador y la interacción con actores pertenecientes al entorno de la firma (Becheikh, Landry y Amara, 2006).

A la par con las fuentes externas de ideas de innovación, se reconoce que el desempeño innovador es afectado por la capacidad interna de la firma para asimilar y replicar el nuevo conocimiento ganado de fuentes externas (Tsai, 2001). Esta característica ha sido denominada capacidad de absorción, término definido por Cohen y Levinthal (1989, p. 589) como "la habilidad de la firma de identificar, asimilar y explotar conocimiento de su entorno". El grado de colaboración, entendido como la capacidad de las unidades para procesar información, comunicarse y transferir conocimiento (Tyler, 2001), es una de las características que se asocia recurrentemente con capacidad de absorción. La evidencia empírica demuestra que el desarrollo o la explotación de oportunidades o de conocimiento externo es incrementado por la comunicación interna entre subunidades de la firma (Van de Bosch, Volberda y De Boer, 1999). En consecuencia, es de esperarse que en los sectores donde, en promedio, las firmas posean mayor capacidad de transferencia interna de conocimiento, la existencia de un mayor número de fuentes de innovación evidencia un mayor impacto sobre el desempeño innovador medido como gasto en I\&D.

En el caso de registro de patentes, la combinación de la existencia de muchas fuentes externas de ideas para la innovación (redes) (Powell y Grodal, 2005) y el régimen de propiedad débil que posee Colombia (Schwab, 2010) pueden llevar a las firmas a usar mecanismos alternos (v.gr. secreto industrial) a las patentes para proteger las rentas provenientes de las innovaciones. Adicionalmente, si se reconoce que en Colombia los procesos de innovación se basan principalmente en la imitación, adaptación e ingeniería inversa (Forero, Laureiro y Marín, 2007), se espera que las empresas de sectores caracterizados por una alta densidad de intercambio de ideas para la innovación eviten 
proteger sus invenciones radicales por medio de patentes, debido a la develación de información que subyace a este mecanismo.

De acuerdo con los argumentos expuestos, se espera probar que:

Hipótesis 2: 2a) existe una relación positiva entre fuentes externas de ideas para la innovación sectorial y el promedio invertido en IED por sector; 2b) la relación entre fuentes externas de ideas para la innovación sectorial y el promedio invertido en IEDD por sector es moderada positivamente por la capacidad de transferencia interna de conocimiento promedio de las firmas del sector ${ }^{2}$; y 2c) existe una relación negativa entre fuentes externas de ideas para la innovación sectorial y el número de patentes obtenidas por sector.

\subsection{Concentración de la innovación ${ }^{3}$}

Nelson y Winter (1982) distinguen dos tipos de regímenes de innovación industrial: el emprendedor y el rutinizado. El régimen emprendedor se caracteriza por "la destrucción creativa" y un patrón de ampliación de las actividades innovadoras. En este tipo de régimen, las empresas entrantes se basan en el conocimiento científico para crear nuevos mercados que son ignorados por las firmas establecidas (Sarkar, Echambadi, Agarwal y Sen, 2006). De acuerdo con Comanor (1967), los gastos en investigación aumentan la tasa de introducción de productos al mercado, lo cual posteriormente facilitará una mayor diferenciación. Los patrones de innovación son distribuidos por medio de una gran cantidad de empresas, con una alta turbulencia en la actividad innovadora y la aparición frecuente de nuevos innovadores (Malerba, 2007). De acuerdo con lo anterior, y con base en que la actividad innovadora es más intensiva en industrias que se caractericen por baja concentración de la innovación (Damanpour y Aravind, 2006), se esperaría que en estos sectores se evidencie un mayor gasto promedio en I\&D.

Por su parte, el régimen rutinizado representa la "acumulación creativa" y la profundización en un patrón de innovación (Nelson y Winter, 1982). La actividad innovadora es determinada por el stock de conocimientos y experiencias acumuladas de carácter no transferible, pertenecientes a las empresas ya establecidas (Utterback y Abernathy, 1975; Nelson y Winter 1982). En los sectores caracterizados por régimen rutinizado, las

2 Respecto al conteo de patentes, si se reconoce que los procesos de innovación tienen un riesgo de fuga de información asociado debido a la movilidad de científicos, ingenieros y técnicos de la firma (Hall y Lerner, 2010), se podría esperar que, en la medida en que la información acerca del proceso de innovación provenga y sea compartida por más departamentos de la firma, el riesgo de fuga de información sea mayor. En concordancia, la relación entre las redes externas de conocimiento y el conteo de patentes puede ser moderada en forma positiva por la capacidad de transferencia de conocimiento de las unidades de la firma. Consideramos que lo anterior, aunque posiblemente intuitivo, carece de una fundamentación teórica fuerte y de investigaciones que aporten evidencia empírica al respecto, por lo cual nos abstenemos de la formulación de una hipótesis de tal moderación, a pesar de que se hace la exploración estadística al respecto.

3 Dados los variados y a veces contradictorios resultados entre concentración del mercado e indicadores de desempeño innovador (Damanpour y Aravind, 2006), en la presente investigación acogemos la sugerencia de Lee y Noh (2009) acerca del uso de indicadores de concentración del gasto en I\&D por industria. 
actividades de innovación se concentran en pocas firmas, las cuales enfrentan entornos de innovación estables y con poca amenaza de entrada de nuevos innovadores (Malerba, 2007, p. 690-691). La ventaja competitiva de las firmas de estos sectores está basada en el establecimiento de economías de escala, en las capacidades de aprendizaje acumuladas, en el desarrollo de redes de distribución, en la apropiación y protección de las rentas de sus innovaciones anteriores por medio de patentes, así como en los beneficios derivados de la reputación (Sarkar et al., 2006).

En los países emergentes, las innovaciones introducidas por las empresas se deben principalmente a procesos de difusión tecnológica (Lundvall, Joseph, Chaminade y Vang, 2009) y se basan primordialmente en el desarrollo de capacidades tecnológicas y de adaptación de tecnologías provenientes de fuentes externas (Viotti, 2002). Se reconoce, además, que las innovaciones procedentes de países emergentes se obtienen por medio de aprendizajes alternos al generado mediante la inversión en I\&D y que la mayoría de las innovaciones en países emergentes son de bajo contenido de I\&D (Chaminade, Lundvall, Vang y Joseph, 2009). En coherencia con lo expuesto, se podría esperar que, en sectores manufactureros de un país emergente, la baja concentración de las actividades de innovación no necesariamente se relacione en forma positiva con la inversión en I\&D. Adicionalmente, la evidencia empírica demuestra que, a pesar de que la inversión en I\&D de productos se reduce en sectores de alta concentración, la dirigida a la consecución de innovaciones incrementales o mejoras de procesos aumenta (Malerba, 2007; Lee y Noh, 2009). Se espera entonces que, en sectores caracterizados por una alta concentración, el menor gasto en I\&D de productos se compense con un mayor gasto en I\&D dirigido a procesos o innovaciones incrementales.

La posibilidad de patentar depende inicialmente de que las actividades de I\&D que una firma haya emprendido generen innovaciones susceptibles de ser protegidas legalmente, es decir, que sean invenciones. Un requisito para lo anterior es que la firma haya desarrollado capacidades de innovación mediante un proceso complejo y acumulativo de aprendizaje (Bruland y Mowery, 2005). Al respecto, Zhao, Tong, Wong y Zhu (2005) argumentan que un alto grado de desarrollo de la capacidad de innovación se relaciona con la habilidad de la firma para generar conocimiento susceptible de ser protegido por propiedad intelectual.

Respecto al régimen emprendedor, es posible argumentar que las nuevas firmas entran a competir con base en invenciones o patentes; sin embargo, en el caso de Colombia, a pesar de las mejoras evidenciadas en el último lustro, la tasa de emprendimiento por necesidad cuadruplica la evidenciada en países con economías basadas en la innovación (Global Entrepreneurship Monitor [GEM] et al, 2013). Además, la proporción de emprendimientos basados en innovación es baja (GEM et al., 2006), y se observa en estos un amplio uso de tecnologías obsoletas (más de cinco años en el mercado) (GEM et al, 2013). 
Acorde con las características descritas de cada tipo de régimen y según la evidencia de emprendimientos presentada para Colombia, se espera que las firmas localizadas en regímenes rutinizados evidencien mayor acumulación de competencias de innovación que las firmas pertenecientes a regímenes emprendedores. Consecuentemente, se espera observar que, en sectores en los cuales la innovación se concentra en pocas firmas (régimen rutinizado), el conteo de patentes sea mayor que en sectores en los cuales los procesos de innovación son llevados a cabo por muchas firmas (régimen emprendedor).

Además de la capacidad de invención, la decisión de patentar depende de las condiciones de apropiación del sector. Teece (1986) define régimen de apropiación como el conjunto de los factores ambientales que determinan el grado en que una firma puede capturar los beneficios generados por una innovación. Las dimensiones más importantes de este régimen son la naturaleza de la tecnología y la eficacia de los mecanismos jurídicos de protección. Si se tiene en cuenta que, como ya se señaló anteriormente, en Colombia, los procesos de innovación de las SME se fundan principalmente en la imitación, la adaptación y la ingeniería inversa (Forero, Laurerio y Marín, 2007), y que el país posee un régimen de propiedad intelectual débil (Schwab, 2010), se puede esperar que, en industrias en las que exista una gran cantidad de agentes innovadores, el riesgo asociado con la apropiación de rentas sea mayor y que, por lo tanto, en estos sectores el conteo de patentes sea menor que en sectores en los que la innovación se concentra en pocas firmas.

Con base en la argumentación presentada, en la presente investigación se espera probar que:

Hipótesis 3: 3a) la concentración de la innovación se relaciona en forma positiva con el promedio invertido en I\&D por sector y $3 \mathrm{~b}$ ) la concentración de la innovación se relaciona en forma positiva con el número de patentes obtenidas por sector.

\section{Metodología}

En esta sección se describen algunas características y estadísticas descriptivas de las bases de datos usadas para probar las hipótesis propuestas. Adicionalmente, se definen las variables dependientes, las independientes y los controles implementados. Finalmente, se presentan los modelos econométricos estimados.

\subsection{Fuente de datos}

La fuente estadística usada en las estimaciones es la Tercera Encuesta de Desarrollo e Innovación Tecnológica EDIT-III (2005-2006), del DANE el DNP y Colciencias. Esta encuesta tiene información del censo realizado a las 6670 firmas manufactureras nacionales y con la agrupación sectorial asociada con el Código Industrial Internacional Uniforme (CIIU). La 
industria manufacturera del país está constituida por 61 sectores, pero, debido a la ausencia de datos relacionados con las variables usadas en la presente investigación, la muestra real está compuesta por 53 sectores.

\subsection{Variables}

Acorde con los dos indicadores de desempeño innovador que van a ser explorados en la presente investigación, las variables dependientes seleccionadas son el número de patentes obtenidas por sector durante el periodo 2005-2006 (NP) y el monto promedio invertido por el sector en investigación y desarrollo en el periodo 2005-2006 (IPID).

Las variables independientes incluidas en los modelos estadísticos se describen a continuación:

i) Capital extranjero (CE): Número de empresas del sector con $25 \%$ o más del capital extranjero, dividido entre el total de empresas del sector en 2006.

ii) Fuentes externas de ideas para la innovación (FEI): Número de empresas pertenecientes a un sector, cuyas ideas de innovación provienen de al menos un agente externo (clientes, proveedores, competidores, agremiaciones y asociaciones sectoriales, cámaras de comercio, centro de desarrollo tecnológico (CDT) y universidades y/o centros de investigación), dividido entre el total de empresas del sector en 2006.

iii) Capacidad interna de transferencia de conocimiento (GITC): Número de empresas pertenecientes a un sector, cuyas ideas de innovación provienen de al menos un agente interno (departamento interno de I\&D, directivos de la empresa, departamento de producción, departamento externo de I\&D, grupos interdisciplinarios, departamento de ventas y mercadeo y trabajadores), dividido entre el total de empresas del sector en 2006.

iv) Fuentes externas de financiación (FEF): Esta variable mide el acceso del sector a fuentes públicas o privadas de financiación para la innovación. Si el sector no es financiado por la banca privada ni por la pública, se le asigna cero como puntaje; si el sector es financiado por al menos una de estas fuentes, se le asigna un puntaje de 1 ; y si el sector es financiado por la banca privada y por la pública, se le asigna un puntaje de 2 .

v) Concentración de la innovación (CI): Monto invertido en innovación y desarrollo por las grandes empresas del sector, dividido entre el monto total invertido en innovación y desarrollo por el sector.

vi) Oportunidad tecnológica (OT): Se considera que los sectores caracterizados por alto conocimiento proporcionan mayor oportunidad tecnológica que los de bajo conocimiento (Bhattacharya y Bloch, 2004). La clasificación de los sectores de la industria manufacturera colombiana se hizo a partir de la agrupación propuesta por 
Gera y Massé (1996). Estos autores clasifican los sectores industriales de acuerdo con una combinación de indicadores de investigación y desarrollo (I\&D) e identifican tres categorías: altas, medias y bajas. En esta investigación se usó una variable dicótoma que diferencia los sectores cuya base de conocimiento tecnológico es alta de los sectores considerados de medio o bajo conocimiento.

vii) Nivel educativo del capital humano: Personal ocupado en las empresas industriales que contribuyen en el desarrollo de las actividades dirigidas a la innovación tecnológica y el desarrollo. Esta variable se divide en dos: la primera es la fracción de ocupados por las empresas del sector y cuyo nivel educativo es técnico (FET); la segunda es la fracción de ocupados por las empresas del sector y cuyo nivel educativo es de profesional a doctorado (FEPD).

\subsection{Modelos estadísticos}

Para la variable continua inversión promedio en IED, se usó una regresión de mínimos cuadrados ordinarios (MCO). Para la variable de conteo número de patentes se estimó un Modelo Poisson. Se estandarizaron todas las variables, excepto las de tipo dummy. Las interacciones propuestas se obtuvieron de la multiplicación de la variable independiente correspondiente por su moderadora, ambas estandarizadas. En ningún caso los modelos tienen un VIF superior a 5, con lo que se excluyen problemas de multicolinealidad.

De acuerdo con la tabla 1, la variable dependiente registro de patentes por sector presenta una distribución Poisson. Los estadísticos descriptivos (tabla 1) muestran que existe una sobredispersión de los datos (varianza mayor que la media) en esta variable de conteo y un alto porcentaje de ceros en la distribución. Esto genera que el Modelo Poisson deje de ser en sí mismo el mejor modelo. No obstante, en la práctica, las variables de conteo presentan frecuentemente sobredispersión: en estos casos, aunque los estimadores no serán de varianza mínima, si serán consistentes (Long, 1997).

Tabla 1. Estadísticos descriptivos de las variables dependientes

\begin{tabular}{|l|c|c|}
\hline \multicolumn{1}{|l|}{ Variable } & IPID & NP \\
\hline Media & $2.38 \mathrm{e}-09$ & 0.7540984 \\
\hline Varianza & 1 & 5.788525 \\
\hline Desviación estándar & 1 & 2.405935 \\
\hline Valor máximo & 5.976823 & 11 \\
\hline Valor mínimo & -0.3202016 & 0 \\
\hline Curtosis & 24.40114 & 14.96355 \\
\hline
\end{tabular}




\begin{tabular}{|l|c|c|}
\hline \multicolumn{1}{|c|}{ Variable } & IPID & NP \\
\hline Datos perdidos & 0 & 0 \\
\hline Valores negativos & 52 & 0 \\
\hline Valores cero & 0 & 51 \\
\hline Valores positivos & 9 & 10 \\
\hline Observaciones de análisis & 61 & 61 \\
\hline
\end{tabular}

Fuente. Cálculos basados en EDIT (III).

Para la regresión de mínimos cuadrados ordinarios (MCO), además de la prueba de multicolinealidad ya reportada, se probó la no existencia de heterocedasticidad por medio del Test Breusch-Pagan/Cook-Weisberg. Adicionalmente, se probó la normalidad de la distribución del término de error por medio del Test Estadístico Smirnov Kolmogorov; el valor de la prueba (0.55) no es significativo al $1 \%, 5 \%$ y $10 \%$, lo que implica que no se puede rechazar la hipótesis nula de que "los residuos se distribuyen normalmente".

\section{Resultados y discusión}

Los resultados de la estimación de los modelos se presentan en la tabla 3. La primera columna corresponde al Modelo de Regresión Lineal, mientras que la segunda corresponde al Modelo de Regresión Poisson. En la tabla 2 se observan las correlaciones y desviaciones estándares de cada una de las variables incluidas en los modelos mencionados.

Tabla 2. Matriz de correlaciones de las variables utilizadas en el modelo

\begin{tabular}{|c|c|c|c|c|c|c|c|c|c|c|}
\hline $\begin{array}{l}\text { Fuente. EDIT } \\
\text { (III). Variable }\end{array}$ & IPID & NP & GE & FEI & GITG & FEF & GI & OT & FET & FEPD \\
\hline IPID & 1 & & & & & & & & & \\
\hline NP & 0.0248 & 1 & & & & & & & & \\
\hline $\mathrm{CE}$ & 0.0048 & $0.4571^{*}$ & 1 & & & & & & & \\
\hline FEI & 0.2428 & -0.0624 & 0.1155 & 1 & & & & & & \\
\hline CITG & 0.1466 & -0.0509 & 0.1053 & $0.7082^{*}$ & 1 & & & & & \\
\hline FEF & 0.0386 & 0.1724 & $0.3340^{*}$ & 0.1907 & 0.1119 & 1 & & & & \\
\hline CI & 0.1652 & 0.2237 & -0.0265 & 0.0200 & -0.2530 & -0.0120 & 1 & & & \\
\hline OT & $0.3517^{*}$ & 0.1204 & $0.3263^{*}$ & $0.3118^{*}$ & $0.2904 *$ & 0.0814 & -0.1769 & 1 & & \\
\hline FET & -0.0593 & -0.1224 & -0.0122 & $0.4098^{*}$ & $0.6232^{*}$ & 0.0268 & -0.1469 & 0.1875 & 1 & \\
\hline FEPD & $0.2751^{*}$ & 0.0424 & $0.2803^{*}$ & 0.2362 & $0.2884 *$ & 0.1659 & -0.0522 & 0.2444 & $0.4139 *$ & 1 \\
\hline
\end{tabular}

Nota: *Significancia al 0.05. Fuente. EDIT III. 
Tabla 3. Resultados modelos estadísticos estimados

\begin{tabular}{|c|c|c|}
\hline & MCO & Poisson \\
\hline Variables independientes & IPID & NP \\
\hline $\mathrm{CE}$ & $-0.292 *(0.169)$ & $0.824 * * *(0.136)$ \\
\hline FEI & $0.139(0.136)$ & $-1.688^{* * * *}(0.486)$ \\
\hline CITC & $0.175(0.200)$ & $1.057(0.795)$ \\
\hline CI & $0.280 * *(0.130)$ & $0.995^{* * * *}(0.197)$ \\
\hline FEF & $-0.247(0.186)$ & $1.329 *(0.792)$ \\
\hline OT & $1.071 * *(0.523)$ & $2.680(1.664)$ \\
\hline FET & $-0.877^{* *}(0.363)$ & $-1.991 * *(1.000)$ \\
\hline FEPD & $0.588 *(0.317)$ & $-0.591(0.580)$ \\
\hline Interacción FEI x CITG & $0.117 *(0.065)$ & $-1.058(0.970)$ \\
\hline Constant & $0.216(0.355)$ & $-4.665^{* * *}(1.398)$ \\
\hline Observations & 53 & 53 \\
\hline R-cuadrado (MCO)/ R-cuadrado Mc Fadden's (Poisson) & 0.481 & 0.458 \\
\hline Adj. R-squared & 0.373 & \\
\hline VIF & 2.04 & 2.04 \\
\hline Robust standard errors in brackets & $* * * \mathrm{p}<0.01$ & $0.05, * \mathrm{p}<0.1$ \\
\hline
\end{tabular}

Fuente. Cálculos de los autores.

Los resultados constatan las relaciones propuestas entre capital extranjero y las variables dependientes en las hipótesis la y lb. La participación de capital extranjero se relaciona en forma negativa con inversión promedio en IED y en forma positiva con el número de patentes. Este resultado contradice la creencia generalizada de que esta clase de firmas son las más activas en el desarrollo de actividades de I\&D en países emergentes (Langebaek y Vásquez, 2007). Adicionalmente, la relación positiva entre capital extranjero y conteo de patentes parece confirmar la estrategia de protección de derechos de propiedad seguida por multinacionales en algunos países en desarrollo, como lo es la del registro local de sus patentes (o de modificaciones de estas) desarrolladas en el país de origen o ya patentadas en otros países (López y Orlicki, 2009). Al respecto, la evidencia empírica de Montobbio (2007) acerca de patentes de países latinoamericanos es coherente con tal afirmación. Se confirma, entonces, la tendencia de las décadas de los ochenta y los noventa descrita por Aboites (2003), en el sentido de que los países latinoamericanos son receptores de flujos de tecnología codificada en forma de patentes. El análisis conjunto de la relación de la variable propiedad extranjera con las dos variables dependientes usadas confirma que las empresas multinacionales seleccionan y distinguen entre países en los cuales invierten en 
I\&D de países en los cuales solo protegen y explotan el conocimiento y las innovaciones desarrolladas desde la casa matriz.

No existe evidencia que respalde la hipótesis 2a, mientras que los resultados confirman la relación propuesta en la hipótesis $2 \mathrm{~b}$. A este respecto, la no significancia de la relación de fuentes externas de ideas para la innovación y la inversión en I\&D, junto con el resultado significativo y positivo de la interacción entre dichas fuentes y la capacidad de transferencia de conocimiento son indicios de que no es suficiente que una firma se ubique en un sector caracterizado por la existencia de muchas redes de innovación, es necesario, además, el desarrollo de la capacidad de absorción. De acuerdo con Hillebrand y Biemans (2004, p. 118), para que una organización genere aprendizaje de fuentes externas, es necesaria la existencia de "cooperación interna (...) para difundir, interpretar, utilizar y para evaluar los nuevos conocimientos adquiridos de los socios externos".

Adicionalmente, los resultados sustentan la relación propuesta en la hipótesis 2c. La relación negativa y significativa entre las redes externas de innovación y el número de patentes registradas por sector puede deberse a dos razones. La primera es que es reconocido que las fuentes de innovación, a la vez que permiten el acceso a ideas nuevas, actúan como canales de transferencia y difusión de conocimiento (Powell y Grodal, 2005). Otra función de las redes que se constituyen en relación con las fuentes de innovación es la institucionalización de prácticas y procedimientos por parte de los integrantes de un campo organizacional (Owen-Smith y Powell, 2008). En consecuencia, en los sectores en los que existen muchas redes externas y en los cuales la práctica de la copia o imitación se ha institucionalizado, se puede esperar que las empresas que patentan se enfrenten a una mayor probabilidad de disminución de apropiación de las posibles rentas provenientes de la inversión en innovación (Cohen, Nelson y Walsh, 2000).

La segunda razón es la débil protección de los derechos de propiedad del país, situación que facilita la imitación (Akiyama y Furukawa, 2009), disminuye la captura de rentas de las inversiones en I\&D por parte de las firmas (Teece, 1986) e impone altos costos relacionados con la defensa de sus derechos sobre la innovación (Nagaoka, Motohashi y Goto, 2010). A pesar de que en la presente investigación no se probó el efecto directo que tienen los diferentes niveles de protección intelectual sectorial sobre el conteo de patentes, sí se tiene en cuenta que la evidencia empírica muestra que una protección rigurosa es conducente a que la inversión en $\mathrm{I} \& \mathrm{D}$ se transforme en o refleje mayor conteo de patentes por país (Crawford, Fabling, Grimes y Bonner, 2007; Danguy, De Rassenfosse y De la Potterie, 2009). Algunos resultados contrastantes relacionados con las variables de control reportados en la presente investigación son indicios del efecto que la débil protección intelectual tiene sobre el registro de patentes. El primer resultado de este tipo es que, a pesar de que en los sectores en los que se presenta alta oportunidad tecnológica se evidencia mayor inversión en $I \& D$, no existe relación significativa entre 
esta variable y el conteo de patentes por sector, como era de esperarse conceptualmente y por la evidencia empírica disponible (Damanpour y Aravind, 2006).

El segundo resultado tiene que ver con la variable nivel educativo. La relación negativa entre fracción de empleados con formación técnica con inversión en I\&D y conteo de patentes podría suponer que es necesario que los sectores posean recurso humano con mayor grado educativo para obtener invenciones o invertir en I\&D. Lo anterior se confirma parcialmente con la evidencia de que existe una relación positiva entre fracción de empleados con grado profesional o doctoral y la inversión en I\&D por sector. No obstante, la relación no significativa entre esta variable y el conteo de patentes puede sugerir, en concordancia con lo propuesto por Moser (2005), que, en algunos sectores, aunque se posea capacidad de generar innovaciones susceptibles de ser patentadas, se usan mecanismos alternos de protección que aumenten el grado de apropiación más allá del que proporciona la protección por medio de patentes.

Finalmente, se confirma la existencia de una relación positiva entre concentración y las dos variables dependientes usadas, lo cual sustenta lo propuesto en las hipótesis 3a y 3b. De acuerdo con la teoría de los regímenes tecnológicos (Nelson y Winter, 1982), un sector exhibe ciclos de dinámica innovadora que lo hace pasar de regímenes rutinizados a emprendedores; es en tal evolución en la que se apalanca la dinámica innovadora de la industria ${ }^{4}$. La relación positiva encontrada entre la concentración de la innovación y la inversión en I\&D sugiere la existencia de estancamiento de la actividad innovadora en el ámbito sectorial. Este resultado es coherente con lo propuesto por Altenburg (2009), quien argumenta que en algunos países emergentes no se presenta la fase de destrucción creativa, en la cual firmas entrantes basadas en algún tipo de innovación desafían a las firmas dominantes y llevan a o hacen que las firmas menos eficientes salgan del mercado. En consecuencia, la transición de tamaño de las firmas o de los movimientos en la distribución de productividad del sector es muy baja (van Biesebroeck, 2005). En el caso de países emergentes, esta situación puede deberse a que las firmas entrantes a los sectores son no innovadoras o que entran a competir con innovaciones incrementales o de fácil imitación (Viotti, 2002), por consiguiente, estas firmas entran con ventajas competitivas similares a las firmas que recientemente han salido del sector (Altenburg, 2009).

\section{Conclusiones}

Esta investigación se propuso como objetivo contrastar los determinantes del esfuerzo innovador y del conteo de patentes de 53 sectores industriales pertenecientes a una economía emergente. Las variables independientes incluidas en la presente investigación fueron las siguientes: 1) número de firmas extranjeras promedio por sector, 2) redes

4 La evidencia empírica de las investigaciones de Malerba y Orsenigo (1996) demuestra que estos patrones shumpeterianos de innovación son relativamente invariantes entre países industrializados. 
externas de conocimiento sectorial, 3) capacidad interna de transferencia de conocimiento y 4) concentración de la inversión en innovación. Los resultados obtenidos permiten afirmar que se constató que las condiciones del desarrollo tecnológico e institucional de Colombia se reflejan en una relación entre estas variables dependientes y los dos indicadores de desempeño innovador, relación distinta de la que se observa en países industrializados. Los aspectos más importantes resultantes de la presente investigación se destacan a continuación.

Se evidenció la existencia de una relación positiva entre la presencia de capital extranjero del sector y el conteo de patentes, a la vez que existe una relación negativa entre esta variable e inversión en $\mathrm{I} \& \mathrm{D}$. El análisis conjunto de este resultado confirma que las empresas multinacionales seleccionan y distinguen entre países en los cuales invierten en I\&D de países en los cuales solo protegen y explotan el conocimiento y las innovaciones desarrolladas desde la casa matriz. Los resultados sugieren que la débil protección de la propiedad intelectual, el bajo grado de desarrollo tecnológico y el tipo de spillover que se presenta en los sectores manufactureros del país no son incentivos para que las empresas de capital extranjero hagan inversiones en I\&D.

La no significancia de la relación de redes externas de innovación y la inversión en I\&D, junto con el resultado significativo y positivo de la interacción entre redes y capacidad de transferencia de conocimiento, indica que no es suficiente que una firma se ubique en un sector caracterizado por la existencia de muchas redes de innovación, sino que es necesario, además, el desarrollo de la capacidad de explotar internamente el conocimiento o la información obtenidos por medio de tales relaciones.

Se constata, además, que las redes de innovación inciden de forma negativa en el conteo de patentes por sector. El anterior resultado puede ser un indicio de que, en sectores en los que existe un gran número de redes de innovación, las empresas se abstienen de patentar sus innovaciones, a la vez que usan mecanismos alternos de protección. Lo anterior se debe a que la existencia extendida de prácticas de imitación o adaptación por parte de las empresas manufactureras del país, combinada con la existencia de muchos canales de transferencia de conocimiento, puede disminuir la posibilidad de apoderarse de las rentas provenientes del desarrollo de innovaciones.

Finalmente, se confirma la existencia de una relación positiva entre concentración de la innovación y las dos variables dependientes usadas. La relación positiva encontrada entre la concentración de la innovación y la inversión en I\&D sugiere la existencia de estancamiento de la actividad innovadora en el ámbito sectorial. Este resultado es coherente con lo propuesto por Altenburg (2009), en el sentido de que en países emergentes no se presenta la fase de destrucción creativa propuesta por Schumpeter. 
Los resultados obtenidos pueden ser un insumo importante para el diseño de política pública en innovación. El bajo aporte del capital extranjero a la inversión en I\&D puede ser observado como una oportunidad que se puede explotar. Para ello, la política de atracción de capital extranjero directo del país debe pasar de un enfoque centrado en la cantidad a uno dirigido a la calidad. En este sentido, es necesario que, en sectores industriales que posean cierto grado de capacidad de absorción ${ }^{5}$, se incentiven la entrada de firmas extranjeras y el desarrollo de alianzas entre estas y empresas locales, que implique el desarrollo de proyectos de I\&D.

Además de la importancia de la transferencia y adaptación del conocimiento y tecnologías de fuentes externas, la política pública debe también propender por incentivar procesos de desarrollo de competencias en innovación a partir de las capacidades de las firmas locales. En coherencia con lo anterior, se debe incentivar la formación de alianzas entre empresas locales para el desarrollo de proyectos de innovación, así como la formación de clústeres de empresas que incentiven la transferencia de conocimiento. Para lo anterior, es necesario el desarrollo de políticas complementarias en educación y acceso al capital financiero. Respecto a la educación, los resultados muestran la necesidad de enfatizar en la formación de personal técnico altamente especializado, así como mayor cantidad de ingenieros que permitan el desarrollo de competencias innovadoras alternas a la inversión en I\&D. Adicionalmente, para impulsar los sectores empresariales, es necesario el desarrollo de la industria de capitales de riesgo y facilitar el acceso a capital financiero por parte de empresas innovadoras y proyectos emprendedores de base tecnológica.

Finalmente, se requiere el rediseño de instituciones que impidan una mayor fragmentación del sistema de innovación del país. Es indispensable facilitar los procesos de entrada y salida de empresas a los sectores manufactureros, de tal forma que, junto con un mayor apoyo a emprendimientos de base tecnológica, se impulse la fase de destrucción creativa schumpeteriana, generalmente ausente en países emergentes. Además, es indispensable fortalecer la protección de los derechos de propiedad intelectual, de tal forma que sus costos de defensa se reduzcan y se generen incentivos de apropiación de rentas ex post a la innovación.

La presente investigación expone dos limitaciones importantes. La primera es que no se contó con datos de ventas y rentabilidad de los sectores, situación que impidió establecer la forma como los resultados económicos afectan la inversión en I\&D y el conteo de patentes por sector. La segunda limitación es que este estudio ofrece las características de un estudio transversal, por lo que no se puede llegar a conclusiones estadísticas en relación con la existencia y dirección de la causalidad.

5 Se ha demostrado que cuando las firmas extranjeras poseen un nivel de competitividad muy superior a las firmas locales, no existen incentivos para que estas últimas inviertan en actividades de innovación (Altenburg, 2009). 
Con base en las limitaciones señaladas, se considera que es necesario el desarrollo de investigaciones en diferentes niveles de análisis, que permita verificar y buscar explicaciones complementarias o substitutas a los resultados obtenidos. Adicionalmente, los resultados de la variable concentración sugieren que es importante hacer un estudio longitudinal acerca de los ciclos de innovación de los sectores manufactureros colombianos. Se requiere, además, probar de manera directa la incidencia de regímenes sectoriales de apropiación sobre la inversión en I\&D y el conteo de patentes.

\section{Referencias}

Aboites, J. (2003). Innovación, patentes y globalización. En J. Aboites y G. Dutrénit (eds.), Innovación, aprendizaje y creación de capacidades tecnológica (pp. 163-206). México: Universidad Autónoma Metropolitana, Unidad Xochimilco.

Akiyama, T., \& Furukawa, Y. (2009). Intellectual property rights and appropriability of innovation. Economics Letters, 103(3), 138-141.

Altenburg, T. (2009). Building inclusive innovation systems in developing countries: Challenges for IS research. En B-A. Lundvall, K. J. Joseph, C. Chaminade \& J. Vang (eds.), Handbook of Innovation Systems and Developing Countries (pp. 33- 56). Cheltenham, UK - Northampton, U.S.A.: Edward Elgar Publishing Limited.

Baldwin J., Gellatly, G., \& Gaudreault, V. (2002). Financing innovation in new small firms: New evidence from Canada (Statistics Canada Analytical Studies Series 11F0019MIE, documento de trabajo n.o 190).

Becheikh, N., Landry, R., \& Amara, N. (2006). Lessons from innovation empirical studies in the manufacturing sector: A systematic review of the literature from 19932003. Technovation, 26(5-6), 644-664.

Bhattacharya, M., \& Bloch, H. (2004). Determinants of innovation. Small Business Economics, 22(2), 155-162.

Blomqvist, K., \& Levy, J. (2006). Collaboration capability. A focal concept in knowledge creation and collaborative innovation in networks. International fournal of Management Concepts and Philosophy, 2(2), 31-48.

Brouwer, E., \& Kleinknecht, A. (1999). Innovative output and a firm propensity to patent. An exploration of CIS micro data. Research Policy, 28(6), 615-624.

Bruland, C., \& Mowery, D. (2005). Innovation through time. EnJ. Fagerberg, D. Mowery \& R. Nelson (eds.), The Oxford Handbook of Innovation (pp. 349 -379). Oxford, UK: Oxford University Press. 
Cantwell, J., \& Piscitello, L. (2005). Recent location of foreign-owned research and development activities by large multinational corporations in the European regions: The role of spillovers and externalities. Regional Studies, 39(1), 1-16.

Cantwell, J., \& Molero, J. (eds.). (2003). Multinational enterprises, innovative strategies and systems of innovation. Cheltenham, UK: Edward Elgar Publishing Limited.

Chaminade, C., Lundvall, B-A., Vang, J., \& Joseph, K. J. (2009). Designing innovation policies for development. En B-A. Lundvall, K. J. Joseph, C. Chaminade \& J. Vang (eds.), Handbook of Innovation Systems and Developing Countries (pp. 360- 379). Cheltenham, UK - Northampton, U. S. A.: Edward Elgar Publishing Limited.

Cimoli, M., Dosi, G., Nelson, R., \& Stiglitz, J. (2009). Institutions and policies in developing economies. En B-A. Lundvall, K. J. Joseph, C. Chaminade \& J. Vang (eds.), Handbook of Innovation Systems and Developing Countries (pp. 337- 359). Cheltenham, UK - Northampton, U. S. A.: Edward Elgar Publishing Limited.

Cohen, W., \& Levinthal, M. (1989). Innovation and learning: The two faces of R\&D. The Economic Fournal, 99(367), 569-596.

Cohen, W., Nelson, R., \& Walsh, J. (2000). Protecting their intellectual assets: Appropriability conditions and why U.S. manufacturing firms patent or not (NBER document de trabajo n.o 7552). Recuperado de http://www.nber.org/papers/w7552

Comanor, W. S. (1967). Market structure, product differentiation, and industrial research. Quarterly Fournal of Economics, 81(4), 639-657.

Crawford, R., Fabling, R., Grimes, A., \& Bonner, N. (2007). National R\&D and patenting: is New Zealand an outlier? New Zealand Economic Papers, 41(1), 69-90.

Damanpour, F., \& Aravind, D. (2006). Product and process innovations: A review of organizational and environmental determinants. En J. Hage \& M. Meeus (Eds.), Innovation Science and Institutional Change (pp. 38-66). New York: Oxford University Press.

DANE-DNP-Colciencias. (2011). Encuesta de Desarrollo e Innovación Tecnológica en la Industria Manufacturera Colombiana-EDIT IV. Bogotá: Autores.

Danguy, J., De Rassenfosse, G., \& Pottelsberghe de la Potterie, B. (2009). The R\&Dpatent relationship: An industry perspective. European Investment Bank Papers, 14(1)

David, P., Yoshikawa, T., Chari, M. D. R., \& Rasheed, A. A. (2006). Strategic investments in Japanese corporations: Do foreign portfolio owners foster underinvestment or appropriate investment? Strategic Management fournal, 27(6), 591-600.

De Rassenfosse, G., \& van Pottelsberghe de la Potterie, B. (2009). A policy insight into the R\&D-patent relationship. Research Policy, 38(5), 779-792. 
Duguet E. and Kabla I. (1998). Appropriation strategy and the motivations to use the patent system in France: an econometric analysis at the firm level. Annales d'Economie et de Statistique, 49/50, 289-327. (Special issue on the economics and econometrics of innovation).

Feinberg S., \& Gupta, A. (2004). Knowledge spillovers and the assignment of R\&D responsibilities to foreign subsidiaries. Strategic Management fournal, 25(8-9), 823-845.

Forero, C., Laureiro, D., \& Marín, A. (2007). Innovation patterns and intellectual property in SMEs of a developing country. Innovar, 21(42). Recuperado de http://www. scielo.org.co/scielo.php?script=sci_arttext\&pid=S0121-50512011000400010

Gatignon H., \& Anderson, E. (1988). The multinational corporation's degree of control over foreign subsidiaries: An empirical test of a transaction cost explanation. Fournal of Law, Economics, and Organization, 4(2), 305-336.

Geisler, E. (2002). The metrics of technology evaluation: Where we stand and where we should go from here. International Fournal of Technology Management, 24(4), 341-374.

Global Entrepreneurship Monitor (GEM) - Gómez, L., Varela, R., Correales, J. P. y Jiménez, J. E. (2006). GEM Colombia 2006: Reporte de resultados. Universidad de los Andes, Universidad ICESI, Universidad del Norte, Pontificia Universidad Javeriana. Recuperado de http://gemcolombia.org/publicaciones/page/3/

Global Entrepreneurship Monitor - Pereira, F. et al. (2013). Reporte GEM Colombia 2011-2012. Universidad de los Andes, Universidad ICESI, Universidad del Norte, Pontificia Universidad Javeriana. Recuperado de http://gemcolombia.org/ publicaciones/page/2/

Gera, S., \& Massé, P. (1996). Employment performance in the knowledge-based economy (Industry Canada Working Paper n.o 14). Recuperado de https://www.ic.gc. ca/eic/site/eas-aes.nsf/vwapj/wp14e.pdf/\$file/wp14e.pdf

Gomes-Cassares B. (1989). Ownership structures of foreign subsidiaries: Theory and evidence. Fournal of Economic Behavior and Organization, 11(1), 1-25.

Green, H. A. J. (1964). Aggregation in economic analysis: An introductory survey. Princeton, N. J.: Princeton University Press.

Hall, B. H., \& Lerner, J. (2010). The financing of R\&D and innovation. En B. Hall \& N. Rosenberg (eds.), Economics of Innovation (pp. 609- 635). Oxford, UK: Elsevier. 
Hillebrand, B., \& Biemans, W. G. (2004). Links between internal and external cooperation in product development: An exploratory study. Fournal of Product Innovation Management, 21(2), 110-122.

Institute for Management Development. (2010). The World Competitiveness Yearbook 2010. Lausanne: IMD.

Langebaek, A y Vásquez, D. (2007). Determinantes de la actividad innovadora en la industria manufacturera colombiana. Borradores de Economía, 433, 1-36.

Lee, C., \& Noh, J. (2009). The relationship between R\&D concentration and industry R\&D intensity: A simple model and some evidence. Economics of Innovation and New Technology, 18(4), 353-368.

Loewe, M., Blume, J., Schönleber, V., Seibert, S., Speer, J., \& Voss, V. (2007). The impact of favoritism on the business climate: A study on wasta in Fordan. Bonn: German Development Institute.

Long, J. (1997). Regression models for categorical and limited dependent variable. London: SAGE Publications Ltd.

López, A., \& Orlicki, E. (2009). Who uses the patent system in developing countries? A study of patent propensities in Argentina, 1992-2001. Research and Information System for Developing Countries, 151.

López-Claros, A., Porter, M., Sala-i-Martin, X., \& Schwab, K. (eds.) (2006). The Global Competitiveness Report 2006-2007: World Economic Forum. New York, USA: Palgrave Macmillan Publishers. Recuperado de http://ev.am/sites/default/files/attachments/pdf/GCR\%202006-2007.pdf

Lundvall, B-A. (2007). National innovations systems: Analytical concept and development tool. Industry and Innovation, 14(1), 95-119.

Lundvall, B-A., Joseph, K. J., Chaminade, C., \& Vang, J. (2009). Innovation system research and developing countries. En B-A. Lundvall, K. J. Joseph, C. Chaminade \& J. Vang (eds.). Handbook of Innovation Systems and Developing Countries (pp. 1-30). Cheltenham, UK - Northampton, U. S. A.: Edward Elgar Publishing Limited.

Lutero, G. (2010). The aggregation problem in its historical perspective: A summary overview. Third Global Conference on Agricultural and Rural Household Statistics. Recuperado de http://www.fao.org/fileadmin/templates/ess/pages/rural/wye_city_group/2010/ May/WYE_2010.4.1_Lutero.pdf

Malerba, F. (2002). Sectoral systems of innovation and production. Research Policy, 31(2), 247-264. 
. (2007). Innovation and the dynamics and evolution of industries: Progress and challenges. International fournal of Industrial Organization, 25(4), 675-699.

Malerba, F., \& Orsenigo, L. (1996). Schumpeterian patterns of innovation are technology-specific. Research Policy, 25(3), 451-478.

Marín, A., \& Arza, V. (2009). The role of multinational corporations in national innovation systems in developing countries. En B-A. Lundvall, K. J. Joseph, C. Chaminade \& J. Vang (eds.). Handbook of Innovation Systems and Developing Countries (pp. 280- 310). Cheltenham, UK - Northampton, U. S. A.: Edward Elgar Publishing Limited.

Marsili, O. (1999). Techological regimes: Theory and evidence. Recuperado de http://www. lem.sssup.it/Dynacom/files/D20_0.pdf

Molero, J. y García, A. (2008). The innovative activity of foreign subsidiaries in the spanish innovation system: An evaluation of their impact from a sectoral taxonomy approach. Technovation, 28(11), 739-757.

Montobbio, F. (2007). Patenting activity in Latin American and Caribbean countries. Geneva: WIPO - ECLAC.

Moser, P. (2005). How do patent laws influence innovation? Evidence from nineteenthcentury world's fairs. American Economic Review, 95(4), 1214-1236.

Nagaoka, S., Motohashi, K., \& Goto, A. (2010). Patent Statistics as an Innovation Indicator. En B. Hall \& N. Rosenberg (eds.), Economics of Innovation (pp. 1084-1127). Oxford, UK: Elsevier.

Narula, R., \& Zanfei, A. (2005). Globalization of innovation. En J., Fagerberg, D., Mowery \& R. Nelson (eds.), The Oxford Handbook of Innovation (pp. 318- 345). Oxford, UK: Oxford University Press.

Nelson, R., \& Winter, S. (1982). An Evolutionary Theory of Economic Change. Cambridge MA: Harvard University Press.

Owen-Smith, J., \& Powell, W. (2008). Networks and institutions. En R., Royston., C., Oliver., C., Sahlin. R. \& Suddaby (eds.), The SAGE Handbook of Organizational Institutionalism (pp. 596- 623). London: SAGE Publications Ltd.

Powell, W., \& Grodal, S. (2005). Networks of innovators. En J., Fagerberg, D., Mowery \& R. Nelson (eds.), The Oxford Handbook of Innovation (pp. 57-85). Oxford, UK: Oxford University Press. 
Romijn, H., \& Albaladejo, M., (2002). Determinants of innovation capability in small electronics and software firms in southeast England. Research Policy, 31(7), 1053-1067.

Sarkar, MB., Echambadi, R., Agarwal, R., \& Sen, B. (2006). The effect of the innovative environment on exit of entrepreneurial firms. Strategic Management fournal, 27(6), 519-539.

Schwab, K. (ed.). (2010). The Global Competitiveness Report 2010-2011: World Economic. Nueva York: Palgrave Macmillan.

Singh, J. (2007). Asymmetry of knowledge spillovers between MNCs and host country firms. Fournal of International Business Studies, 38(5), 764-786.

Teece, D. (1986). Profiting from technological innovation: Implications for integration, collaboration, licensing and public policy. Research Policy 15(6), 285-305.

Tsai, W. (2001). Knowledge transfer in intraorganizational networks: Effects of network position and absorptive capacity on business unit innovation and performance. Academy of Management fournal, 44(5), 996-1004.

Tyler, B. (2001). The complementarity of cooperative and technological competencies. A resource-based perspective. Fournal of English Technology Management, 18(1), pp.1-27.

Utterback, J. M., \& Abernathy, W. J. (1975). A dynamic model of process and product innovation. OMEGA, 3(6), 639-656.

Van Biesebroeck, J. (2005). Firm size matters: Growth and productivity growth in African manufacturing. Economic Development and Cultural Change, 53(3), 545-84.

Van den Bosch, F. A. J., Volberda, H. W., \& De Boer, M. (1999). Coevolution of firm absorptive capacity and knowledge environment: Organizational forms and combinative capabilities. Organization Science, 10(5) 551-568.

Vega, J., Gutiérrez, A., Fernández, I. y Manjarrés, L. (2008). The effect of external and internal factors on firms' product innovation. Research Policy, 37(4), 616-632.

Viotti, E. B. (2002). National learning systems: A new approach on technological changes in late industrializing economies and evidences from the cases of Brazil and South Korea. Technological Forecasting and Social Change, 69(7), 653- 680.

Zhao, H., Tong, X., Wong, P. K., \& Zhu, J. (2005). Types of technology sourcing and innovative capability: An exploratory study of Singapore manufacturing firms. Fournal of High Technology Management Research, 16(2), 209-224. 